\section{La investigación tecnológica en el Deming}

\section{Technology research in Deming}

\section{Fabiola Cevallos Veintimilla*}

\section{RESUMEN}

Los países con mayor capacidad de investigar y generar conocimientos logran un mayor desarrollo económico y social en este mundo globalizado. La importancia que se concede al conocimiento científico y tecnológico como un marcador de desarrollo socioeconómico es creciente, el mismo que requiere de una infraestructura científica sólida, apoyo económico y político del Estado. El presente artículo recoge los resultados de la investigación del uso y manejo de la tecnología y la innovación en el Instituto Superior Tecnológico Corporativo Edwards Deming en el período 2019-2020, para de esta manera formar profesionales que aporten conocimientos, ideas, inventos e innovaciones. Se utiliza la investigación tecnológica que se define como útil para el apoyo y resolución de problemas, orientada a desarrollar una sociedad mejor, a través de procesos activos o convenciones tecnológicas, comprende la descripción, registro, análisis e interpretación de la naturaleza actual y la composición o procesos de los fenómenos; el enfoque se hace sobre conclusiones dominantes o sobre cómo una persona, grupo o cosa funciona en el presente; El Ecuador requiere serias y profundas transformaciones; por lo que es urgente incrementar la eficiencia en el empleo de la tecnología e innovación importada y desarrolle la propia.

Palabras clave: desarrollo investigación, innovación tecnología
REVISTATECNOLÓGICA ciencia y educación Edwards Deming

ISSN: 2600-5867
Atribución/Reconocimiento-NoCo mercial- Compartirlgual 4.0 Licencia Pública Internacional - CC

\section{BY-NC-SA 4.0}

https://creativecommons.org/licenses /by-nc- sa/4.0/legalcode.es

Editado por: Tecnológico Superior Corporativo Edwards Deming Enero - Julio Vol. 5 - I - 202। https://revista-edwardsdeming.com/index.php/es e-ISSN: 2576-097I

Recibido: 14 Marzo 2020

Aprobado: 4 Diciembre, 2020

Pag 1-12

*Master, Instituto Tecnológico Superior Corporativo Edwards Deming, Quito, Ecuador, fabicev@hotmail.com https://orcid.org/0000-0002-4I88204I 


\begin{abstract}
Countries with greater capacity to research and generate knowledge achieve greater economic and social development in this globalized world. The importance attached to scientific and technological knowledge as a marker of socio-economic development is growing, which requires a solid scientific infrastructure, economic and political support from the State. This article collects the results of the research of the use and management of technology and innovation at the Edwards Deming Higher Corporate Technological Institute in the period $2019-2020$, in order to train professionals who contribute knowledge, ideas, inventions and innovations. It uses technological research that can be defined as useful for the support and resolution of problems, aimed at developing a better society, through active processes or technological conventions, comprises the description, recording, analysis and interpretation of the current nature and composition or processes of phenomena; the focus is on dominant conclusions or on how a person, group or thing works in the present; Ecuador requires serious and profound transformations; therefore, it is urgent to increase the efficiency in the use of imported technology and innovation and develop one's own.
\end{abstract}

Keywords: research development, technology innovation

\title{
INTRODUCCIÓN
}

En la sociedad actual, la investigación es una exigencia académica no sólo por el hecho de ser parte de la naturaleza misma de las instituciones de educación superior, sino también por la responsabilidad social ligada al quehacer educativo de los Institutos Técnicos-Tecnológicos.

El Instituto Superior Tecnológico Corporativo Edwards Deming, por su parte, incursiona en un proyecto de investigación sobre el estado situacional de la incorporación de la tecnología en los procesos de aprendizaje en el período 2019 - 2020, utilizando el método descriptivo y la técnica de la observación, una vez que cumple con la responsabilidad de facilitar e impulsar la incorporación a la sociedad del conocimiento para alcanzar los objetivos del régimen de desarrollo; Promover la generación y producción de conocimiento, fomentar la investigación científica y tecnológica y potenciar los saberes ancestrales, para así contribuir a la realización del buen vivir, al Sumak Kausay; asegurar la difusión y el acceso a los conocimientos científicos y tecnológicos, el usufructo de sus descubrimientos y hallazgos en el marco de lo establecido en la Constitución y la ley". 
Por otra parte, también se garantiza la libertad de creación e investigación en el marco de respeto a la ética, la naturaleza, el ambiente y el rescate de los conocimientos ancestrales, además de reconocer la condición de investigador de acuerdo con la Ley. Una de las políticas del Plan Nacional de Desarrollo 2018 - 2022 del Estado Ecuatoriano es Promover la Investigación Científica y la Innovación Tecnológica para propiciar procesos sostenibles de desarrollo. Las principales estrategias de esta política es el incremento de la inversión en Ciencia y Tecnología; promoción de procesos sostenidos de formación académica de investigadores/as; fomento de procesos sostenidos de articulación entre los sectores académicos, gubernamental y productivo; fortalecimiento del Sistema Nacional de Ciencia y Tecnología.

Este desarrollo tecnológico trajo consigo un cambio de mentalidad y por ende una transición a la estructura misma del ámbito educativo, las personas ya se preparan para la era digital tan utilizada en las diferentes áreas laborales, científicas y creativas, que antes no podían soñarse.

\section{MATERIALES Y MÉTODOS}

El presente artículo se extrae del trabajo de investigación realizado por el Instituto Edwards Deming en el período 2019 - 2020. Según Jonathan Llamas 2.020, expresa que la investigación tecnológica consiste en la búsqueda del conocimiento que se pueda definir como útil para el apoyo y resolución de problemas, orientados a desarrollar una sociedad mejor, perfeccionando procesos, activos o convenciones tecnológicas.

En cuanto al nivel de conocimiento es una investigación descriptiva ya que interpreta la situación actual. Para Sabino (2012) los trabajos de indagación suelen clasificarse en aplicados, según su propósito de vinculación a la resolución de un problema práctico y es en la investigación aplicada, donde los conocimientos a obtener son el insumo necesario para proceder a la acción

Según Tamayo y Tamayo (2006), el tipo de investigación descriptiva comprende la descripción, registro, análisis e interpretación de la naturaleza actual y la composición o procesos de los fenómenos; el enfoque se hace sobre conclusiones dominantes o sobre como una persona, grupo, cosa funciona en el presente; la investigación trabaja sobre realidades de hecho caracterizándose fundamentalmente por presentar una interpretación correcta.

Cuando se plantean ideas de progreso y búsquedas de nuevos métodos siempre se asocia la innovación, este proceso se da cuando se quiere mejorar algo que ya existe, dar solución a un problema o facilitar una actividad.

Según el mismo autor, Innovación es una acción de cambio que supone una novedad. Esta palabra procede del latín innovatioo, -ōnis que a su vez se deriva del término innovo, -are "hacer nuevo", "renovar", que se forma con in- "hacia dentro" y novus "nuevo".

Parafraseando a Jonnathan Llamas la innovación se evidencia a través de una acción continua en los diferentes campos del desarrollo humano y no suele aparecer de manera inmediata; más bien ocupa largo tiempo. Algunos sinónimos que suela aparecer son adelanto, invento, reforma, renovación, entre otros. Algunos de los ejemplos de 
innovación tecnológica que se pueden mencionar están los diversos dispositivos electrónicos, como los teléfonos móviles de última generación que contienen diversas aplicaciones útiles para los usuarios.

Según "Innovación" 2018. Disponible en: https://www.significados.com/innovacion/ se toma en cuenta los diferentes aspectos que se han tratado para el tema de innovación: Innovación empresarial.- En el mundo empresarial la innovación es uno de los elementos que se tienen en cuenta a la hora de tener éxito comercial. El concepto de innovación empresarial puede hacer referencia a la introducción de nuevos productos o servicios en el mercado y también a la organización y gestión de una empresa. En ocasiones los productos o servicios comercializados no suponen un cambio en sí, ya que la novedad puede consistir en un nuevo enfoque a productos ya existentes de acuerdo a las demandas del mercado.

Innovación educativa.- supone introducir cambios novedosos en esta área para mejorar el proceso de enseñanza y aprendizaje a través de los materiales utilizados, por ejemplo, la introducción de dispositivos interactivos como las pizarras digitales en el aula; así como a las actividades, la temporalización o los métodos de evaluación.

En ocasiones, los cambios que se introducen afectan a todo el proceso educativo. Por ejemplo, los procesos formativos de educación a distancia suponen una innovación educativa que conllevan cambios a todos los niveles,

El concepto de "innovación disruptiva" se utiliza especialmente en el área empresarial. Se refiere un proceso de cambio innovador orientado a un público minoritario y que se convierte con rapidez en una realidad con gran demanda comercial, se da cuando nuevas empresas presentan nuevos productos, servicios o modelos de negocio que superan en el mercado a empresas líderes del mismo sector. Un ejemplo puede ser Skype, que supuso una innovación y un éxito en el mercado de las telecomunicaciones.

En muchos casos, la innovación está fuertemente unida a la creatividad, el descubrimiento y la invención. Para realizar un cambio que suponga introducir algo nuevo es necesario un proceso creativo.

En ocasiones, la creatividad representa una variación de algo ya existente, por ejemplo, mediante una asociación de ideas. Los conceptos de innovación y creatividad se dan en diversos ámbitos como en la industria, la empresa, la educación y el arte.

La innovación puede darse introduciendo elementos novedosos en la solución o bien, reorganizando de otra forma elementos ya existentes. Decimos pues que no siempre se da en la investigación porque la búsqueda de conocimiento útil también se puede dar reutilizando elementos en el mismo orden para otros problemas.

Un ejemplo es la utilización del teléfono móvil a modo de cámara fotográfica. No supone una innovación novedosa (puesto que existen cámaras fotográficas) ni una reorganización de elementos. Es decir, supone una inclusión de un elemento ya existente dentro de otro también ya existente.

Las investigaciones tecnológicas y las científicas tienen en común varios factores, como pueden ser la meta del conocimiento y los métodos básicos por lo que se rige: hipótesis, datos, interpretación y resultado. Pero ambas investigaciones también poseen varias 
diferencias importantes que se debe destacar. Por una parte, la investigación tecnológica posee una orientación puramente práctica y útil, lo que derivará en un resultado en la mayoría de las ocasiones tangible

Por otra parte la investigación científica se dedica a la búsqueda del conocimiento sin discriminación, los cuales pueden no poseer una aplicación práctica y casi siempre suponen un conjunto de teorías. Estas bien utilizadas, podrían llegar a ser de utilidad en futuras investigaciones tecnológicas.

En este trabajo se usó la técnica de observación que según Ander-Egg, es el procedimiento empírico por excelencia. ... Mediante la observación se intentan captar aquellos aspectos que son más significativos de cara al problema a investigar para recopilar los datos que se estiman convenientes, la aplicación de esta técnica permitió recoger información que se analizó con la perspectiva y los conocimientos previos del observador. Es un método de percepción que permite obtener información del mundo. A continuación se presenta el sistema incorporado en el Deming, mismo que se procedió al proceso de observación de su funcionamiento en cada aspecto relacionado a lo académico.

\section{RESULTADOS}

\section{SISTEMA ACADÉMICO BI-ACADEMOS}

EI SISTEMA ACADÉMICO BI-ACADEMOS, desarrollado por Binary Sistemas, es un sistema modular y parametrizable, el mismo que permite llevar un correcto registro de las actividades académicas de las instituciones de educación superior. Está desarrollado en un ambiente WEB, el mismo que brinda enormes ventajas de accesibilidad y control. Este software ha sido creado a partir de la necesidad de contar con un sistema de información real y oportuna tanto de la parte académica como del aspecto financiero.

BI-ACADEMOS ha sido probado y ha tenido excelentes resultados respecto a su rendimiento y usabilidad. Se puede acceder a él desde cualquier dispositivo que tenga conexión a internet, incluso Smartphone pues está construido bajo tecnología responsiva que adapta el contenido del aplicativo al tamaño del dispositivo desde el cual se está conectando.

Los módulos disponibles en BI-ACADEMOS son los siguientes:

I. Módulo de Clientes

2. Módulo Académico (Autoridad - Docente - Estudiante)

3. Módulo de Reportes

4. Módulo de Facturación (control de pagos de los alumnos)

5. Módulo de Caja

6. Módulo de Configuración

7. Módulo de Titulación (Prácticas Pre profesionales - Vinculación Titulación)

8. Módulo de Comunicaciones

9. Módulo de Bolsa de Trabajo 
Características: fácil, personalizable, rápido, ilimitado seguro Toda actividad está almacenada en el sistema, cumple con todos los requerimientos internacionales respecto a seguridad y confidencialidad de la información. Se Cuenta con servicios de alojamiento con un $\mathbf{9 9 . 9 9 \%}$ de efectividad en línea. Nuestros servidores se encuentran físicamente localizados en Orem, UTAH, Estados Unidos de Norteamérica. Además, contamos con dos (2) tipos de respaldo (backups) de la información: Uno automático (cada mes) y otro manual (cada semana).

\section{DESCRIPCIÓN FUNCIONAL}

MÓDULO DE CLIENTES. - permite la administración de los datos personales, generales, familiares, de contacto y de historial académico de todos los entes creados en el sistema.

Creación rápida de persona natural.- Esta opción permite el registro en la base de datos del sistema de personas naturales, se requiere datos básicos.

Creación de estudiantes.- Esta opción permite registrar los datos completos de los futuros estudiantes, es un requisito previo a la matrícula y la información detallada en éste registro permite formar la estructura de información de estudiantes matriculados que se envía a los organismos de control.

Creación de docentes.- Esta opción permite registrar los datos completos de los docentes.

\section{MÓDULO ACADÉMICO}

En este módulo se generan y administran todos los procesos académicos del Instituto, desde la creación de una carrera, hasta el registro de las calificaciones de los estudiantes. Es el módulo más extenso en opciones y funcionalidad, configuran los datos esenciales del módulo, por lo tanto es el de mayor importancia.

Configuración.- En esta opción se crean:

- Grupos de carrera

- Tipos de carrera

- Modalidad de estudio

- $\quad$ Sistemas de estudio

- $\quad$ Extensiones o Sedes

- Áreas de estudio

- Crear o Activar periodo académico

- Crear grupos de estudiantes

- Activar/Desactivar etapa de calificaciones

- Importar archivo de estudiantes

- Texto anexos y contratos

- Texto mensaje calificaciones estudiantes

Crear Carrera.- Permite registrar los datos necesarios para crear una carrera en el sistema. 
Buscar Carrera.- Opción que permite realizar la consulta y edición de una carrera previamente creada.

Crear Materia.- Permite registrar los datos necesarios para crear una materia y vincularla a una carrera previamente creada, es aquí donde se asigna la malla curricular a la carrera, los créditos u horas de cada materia y el costo de la misma.

Buscar Materia.- Opción que permite realizar la búsqueda y edición de una materia, así como la asignación de pre requisitos en materias que son de segundo nivel en adelante. Adicionalmente permite desactivar materias que por algún cambio en la malla ya no formen parte de la misma, sin que éste hecho afecte el histórico de los estudiantes que si tomaron dicha materia.

Sistema de Calificación.- Esta opción sirve para parametrizar la metodología de calificación a usar en el periodo académico en curso, permite la creación de componentes específicos y su valoración frente a la calificación final, esto permite crear una planilla de calificaciones específica para cada carrera y/o modalidad de estudio.

Docente Materia.- En esta opción se asignan los docentes a cada materia que dictan, de acuerdo a sus competencias y aptitudes. También se usa para la reasignación de docentes cuando existen cambios de docente a algún grupo de estudiantes previamente asignado.

Inscripción.- Opción en la cual se registra la inscripción de estudiante, se asigna la carrera de estudio, este proceso se realiza una sola vez por cada estudiante. En caso de que la institución realice un proceso de inducción previo al inicio de una carrera estudiantil, el acto de ejecutar una inscripción puede contener un componente financiero (rubro) que será parte de la tabla de pagos del estudiante.

Matrícula.- Es una pantalla que tiene varias funcionalidades, la principal, registrar de manera formal la matrícula de los estudiantes, asignando la carrera, la sede, la modalidad de estudio, el nivel (automático), el grupo, las materias a tomar (automático). La opción cuenta con varias validaciones y sub procesos académicos y financieros, mismo que se detalla a continuación:

- Controla el tipo de matrícula (normal, extraordinaria y especial)

- Asigna al módulo financiero la carga financiera de acuerdo al número de materias que el estudiante toma en el periodo académico y en base al tipo de matrícula, así como si se trata de una materia normal o de arrastre y permite asignar un plan de pagos en base al requerimiento del estudiante.

- Controla de manera automática el nivel en el cual se matrícula cada estudiante y en base a la malla determina si existen materias de arrastre, es decir genera un control lógico en el avance académico del estudiante.

- Control que el estudiante no tome más materias que sumen más allá del número de créditos y horas de estudio permitido.

- Genera de manera automática documentos, contratos, anexos y certificados de matrícula.

- Genera el usuario y clave de acceso al sistema y lo envía por mail al correo electrónico 
registrado por el estudiante al momento de la matrícula.

Convalidar/Homologar.- Opción que se usa para registrar las calificaciones de las materias que los estudiantes han obtenido como aprobadas mediante procesos de homologación de malla curricular o por convalidación de conocimientos.

Calificar.- Es la opción que permite a los docentes registrar las calificaciones obtenidas por los grupos de estudiantes asignados a su catedra, la planilla de calificaciones se estructura en base a los parámetros definidos en la pantalla de Sistema de Calificación. Es aquí donde se determina si un estudiante aprueba, se queda en supletorio o reprueba una materia.

Toda vez que se completan los componentes y etapas de evaluación previamente definidas en la pantalla Sistema de Calificación de manera automática el sistema asigna los estados finales a las materias, en caso de que el estudiante se encuentre en estado supletorio, deberá rendir el examen supletorio, el docente registrará la calificación obtenida en el casillero destinado para el efecto y el sistema realizará un recálculo para determinar si el estudiante aprueba o pierde la materia.

Anular Matrícula.- Esta opción nos permite realizar el proceso de anulación de matrícula de un estudiante, tiene como validación que el estudiante no tenga notas asentadas y que no hayan transcurrido más de 31 día del inicio de clases.

Retirar Estudiante.- Pantalla mediante la cual, el usuario autorizado registra en el sistema el estado de retirado de un estudiante, este acto tiene dos acciones en sistema:

I. Académicamente realiza un cálculo de las calificaciones obtenidas hasta el momento del retiro, así como de la asistencia y asigna estados finales a las materias (Aprobado o Perdido).

2. Financieramente suspende el cobro de los haberes o cuotas que registre como pendientes en el periodo académico que se encuentra cursando.

Historial de Calificaciones.- Esta opción se usa cuando la institución hace la implementación del sistema y sirve para registrar las calificaciones de los niveles anteriores a la obtención del sistema, es decir las calificaciones obtenidas por los estudiantes en los periodos académicos que la institución no contaba con el sistema BiAcademos.

Gestión de Becas.- Se utiliza para la creación, actualización y desactivación de las becas que la institución otorga a los estudiantes, se debe registrar el nombre de la beca, el porcentaje de descuento y el o los rubros a los que aplica dicho porcentaje (Matrícula, Pensión, Arrastre).

Esta opción se complementa con la que detallamos en el módulo de clientes que es donde se le asigna la beca al estudiante y este acto genera que el descuento se calcule de manera automática en la tabla de amortización de pagos o cuotas del estudiante.

Cambio de Carrera.- Esta opción permite al personal encargado de la dirección académica de la institución, registrar el requerimiento del estudiante para realizar un cambio de carrera interno en la institución, al ejecutar el proceso se emite un documento de solicitud del cambio con un detalle de todas las materias aprobadas en la 
carrera actual y permite que el usuario matriculador pueda registrar la matrícula del estudiante en la nueva carrera, el documento servirá para realizar el proceso de homologación de materias en la nueva carrera.

Sílabos y Tareas.- Es un submódulo que permite cargar al docente el sílabo que revisará con sus estudiantes, de igual manera permite al estudiante descargarlo y revisarlo.

También permite al docente generar tareas (una $\circ$ varias) para sus estudiantes y asignarles fecha máxima de entrega, las tareas pueden ir dirigidas a todo el grupo de estudiantes $\circ$ un estudiante en específico, el estudiante deberá responder la tarea mediante la carga de un archivo o un audio o video según lo haya determinado el docente, una vez respondida la tarea el docente puede revisar el contenido cargado por el estudiante y asignarle una calificación y un comentario, de la misma manera el estudiante podrá revisar la calificación obtenida y revisar la observación realizada por el docente.

El docente puede de la misma manera y bajo la misma mecánica generar un proyecto, cuyo peso en la calificación de la etapa evaluada es más alta que el de una tarea.

El docente también podrá generar actividades, la metodología tiene un pequeño cambio si lo comparamos con tareas o proyecto, y es que las actividades no requieren de una respuesta del estudiante, sino que el docente evalúa el cumplimiento de la actividad (lección oral, exposición, actividad o aporte en clase) y registra la calificación.

El sistema va realizando un promedio automático de todas las tareas, proyectos y actividades que se han generado en el submódulo y dicho promedio lo registra en la planilla general de calificaciones en el casillero de componente que se defina por la institución.

El estudiante puede responder las tareas y los proyectos hasta que el docente le haya evaluado o hasta que se cumpla la fecha máxima, el docente puede otorgar prórrogas.

Guías Académicas.- Es una opción que se usa para la carga de guías académicas, normalmente lo realiza la dirección académica, de igual manera el estudiante podrá realizar la consulta o descarga de la guía académica de cada materia en la que se encuentre matriculado.

Justificar Asistencia.- Esta opción se usa para que el estudiante registre la solicitud de justificación de una falta a sus clases, el estudiante deberá registrar la materia, la fecha, el motivo de la inasistencia y adjuntar un documento que lo certifique.

Esto genera una tarea para el encargado (docente o autoridad), quien podrá aprobar o negar la justificación.

Cierre de Calificación.- Es una opción que se vincula a la planificación, se llena de manera automática cuando la administración registra la planificación semestral y sirve para controlar la fecha de cierre de la planilla de calificaciones para los decentes, permite llevar un control estricto del cumplimiento de la planificación y el calendario académico, también permite que el usuario administrador amplíe la fecha de cierre cuando existe una solitud del docente. Deja un log de cambio que se usa para el cálculo de índices de cumplimiento de planificación y eficiencia del docente. 
Bloqueo Materia Estudiante.- Es una opción que se usa para restringir el acceso del estuante al contenido de una materia específica.

Planificación.- Es una opción que permite al administrador generar la planificación semestral, registrando los datos de docente asignado al grupo estudiantil, a la materia, las fechas de clases o tutorías, apertura del contenido de la materia, cierre de la materia, examen, supletorio.

MÓDULO DE TITULACIÓN.- Una vez que los estudiantes han concluido con éxito la fase de materias de la malla curricular, inicia la fase de titulación, en este módulo los funcionarios de la institución y los estudiantes podrán ejecutar todos los procesos necesarios para poder graduarse.

Práctica pre profesional y Vinculación con la sociedad.- En esta opción el funcionario responsable podrá visualizar el detalle de estudiantes matriculados desde tercer nivel para iniciar el proceso y llegar a su consecución

Egresados.- Aquellos estudiantes que tengan cumplidos los requisitos y adicionalmente cuenten con las autorizaciones de las áreas: Financiera, Biblioteca y Bienestar Estudiantil podrán iniciar su proceso de titulación, escogiendo la modalidad: Trabajo de Titulación, Proyecto de Emprendimiento o Examen Complexivo.

Calificaciones por alumno.- Permite generar certificados de notas por periodo, certificado consolidado de calificaciones de todas las materias del estudiante, certificado de pase de nivel, detalle de calificaciones del estudiante.

Calificaciones docente.- Permite consultar e imprimir la planilla de calificaciones de los grupos de estudiantes a los cuales dictó cátedra, esta planilla debe ser firmada y entregada a la secretaría académica para el respectivo archivo físico.

Calificaciones generales.- Es un reporte que permite llevar un control del registro de calificaciones de los estudiantes. Constituye una herramienta importante para que la dirección académica identifique a los docentes que no ha realizado el proceso de registro de las calificaciones.

Reporte de planificación.- Es un reporte que permite consultar la planificación de clases por docente.

Cargar listado de egresados.- Es una opción que permite cargar un detalle de personas que pueden realizar la carga de su hoja de vida.

Bolsa de empleo empresas.- Esta opción permite a las empresas registrar una solicitud para poder cargar ofertas laborales dentro de la bolsa de empleo.

El registro de la solicitud genera una tarea para el funcionario del instituto, quien la revisa y autoriza. Si la solicitud es autorizada se genera un usuario y clave para que la empresa pueda acceder a la suit de carga de ofertas laborales.

Estas ofertas son publicadas en la sección de noticias de la web del instituto, donde los estudiantes las pueden visualizar.

Bolsa de empleo estudiantes.- Aquí los estudiantes, interesados en obtener empleo podrán cargar su hoja de vida. 
Sección de noticias de la página web.- En esta sección de la web se publican las ofertas laborales ingresadas por las empresas y permite que los estudiantes que han cargado sus hojas de vida puedan aplicar al empleo.

El empresario analiza la hoja de vida, realiza el proceso de selección y de darse la contratación registra en el sistema que el estudiante o graduado fue contratado.

\section{DISCUSIÓN}

El ámbito educativo se encuentra en un proceso de transición. La sociedad está evolucionando de una mentalidad industrial a un enfoque totalmente informático. La vida cotidiana de las personas está cambiando y se están enfrentando a nuevas herramientas que logran hacer desaparecer los viejos métodos para ejercer una actividad.

En el caso específico de la innovación tecnológico en el Deming, se caracteriza por un sostenido y avance continuo, se caracteriza por ser un proceso de creación de dispositivos nuevos o de modificación de nuevos elementos ya existentes y que mejoran su utilización en los diferentes procesos, que conllevan a la competitividad y desarrollo de bienes y servicios tecnológicos de alta calidad.

Las herramientas tecnológicas se están enfocando en dar solución para al seguimiento del alumnado y sobre todo a que éste pueda aprender y adaptarse a los constantes cambios de tecnología. El emprendimiento ha adquirido un especial protagonismo en este escenario, la activación de la iniciativa emprendedora se plantea como una solución en la educación

Otro aspecto, es que los alumnos ya no tienen por qué seguir el ritmo dictado por el profesor, lo que elimina parte de la tensión del alumno hacia el aprendizaje, de esta manera el alumno desarrolla habilidades y aptitudes a su tiempo, forma y gusto de manera totalmente personalizada. Sencillez, seguimiento y adaptación del usuario, son los aspectos principales en los que la tecnología educativa puede enfocarse para la innovación de nuevas herramientas y a nuevos métodos de trabajar en un futuro.

Se predice que las herramientas de tecnología de la educación encontrarán espacios en la telefonía móvil de un modo mucho mejor al que se encuentran hoy en día. Prohibir estos dispositivos en las aulas será cosa del pasado. La formación de docentes que sean capaces de crear contenidos educativos para el teléfono celular será una necesidad.

El pronóstico es claro: esta clase de herramientas serán un eje central en los métodos de enseñanza. Los beneficios serán numerosos, pero las instituciones educativas tendrán que complementar la inteligencia artificial avanzada en sus modelos de aprendizaje presenciales y la interacción con otras personas.

Los docentes del Deming están actualizando sus saberes disciplinares, tecnológicos, pedagógicos y didácticos a través de experiencias innovadoras de aprendizajes mediadas por las TIC en diferentes contextos educativos diseñan e implementan, de manera crítica, ética y reflexiva, ambientes de aprendizajes creativos, flexibles, motivadores y pertinentes, para promover en sus estudiantes el desarrollo de competencias del siglo XXI. 
Educación que no se enfoque en la memorización sino en el análisis, concentración y toma de decisiones. La tecnología de la educación presenta herramientas sencillas y adaptables a las necesidades del alumnado y a la sociedad dónde se desarrollan.

La coordinación de Investigación Tecnológica, del Instituto Superior Tecnológico Corporativo "Edwards Deming", ha definido las políticas, estrategias y líneas de investigación científica educativa, tecnológica, sostenible e industrial, así como propende la participación, difusión y vinculación con la colectividad a través de publicaciones, talleres, seminarios, congresos, guías de campo, etc., además de capacitación y captación de recursos humanos para la investigación;

El lema utilizado en esta institución es que la piedra fundamental del desarrollo es a través de un manejo de conocimientos ordenados, que actualmente es la base fundamental del desarrollo humano. Solo trabajando en pro de la ciencia, base de la tecnología, podremos solucionar problemas como la miseria, la delincuencia, el subdesarrollo educativo e industrial, el desempleo, entre muchos otros.

\section{REFERENCIAS}

Ander E. Técnicas de observación Departamento de Sociología II. Universidad de Alicante

Barros-Bastidas, C., \& Gebera, O. T. (2020). Training in research and its incidence in the scientific production of teachers in education of a public university of Ecuador. Publicaciones de La Facultad de Educacion y Humanidades Del Campus de Melilla, 50(2), I67-I85. https://doi.org/I0.30827/publicaciones.v50i2.13952

Bunge M. 2006. Las ciencias sociales en discusión. Una perspectiva filosófica. Editorial Sud Americana.

Constitución de la República del Ecuador. 2008 Art. 387

http://www.fisica.uh.cu/biblioteca/revcubfi/redaccioncientifica/articulol.htm.

Recuperado el 12 de noviembre de 2010.

Jonathan LI. (I I de mayo, 202 I). Era digital. Economipedia.com

Plan Nacional de Desarrollo 2018 - 2022

Sabino C. 2012, El Proyecto de la Investigación. $6^{\circ}$ Edición, Editorial Episteme

Tamayo, M. 2006. El Proceso de la Investigación Científica. Limusa. Norega Editores. 